\title{
Targeting the "undruggable" RAS - new strategies - new hope?
}

\author{
Britta Mörchen ${ }^{1,2}$, Oleksandr Shkura ${ }^{3}$, Raphael Stoll ${ }^{3, \#}$, Iris Helfrich ${ }^{1,2, \#}$ \\ 'Skin Cancer Unit of the Dermatology Department, Medical Faculty, University Duisburg-Essen, West German Cancer Center, \\ Essen 45147, Germany. \\ ${ }^{2}$ German Cancer Consortium (DKTK) partner site Düsseldorf/Essen, Essen 45147, Germany. \\ ${ }^{3}$ Biomolecular NMR, Faculty of Chemistry and Biochemistry, Ruhr University of Bochum, Bochum D-44780, Germany. \\ \#Both authors contribute equally.
}

Correspondence to: Dr. Iris Helfrich, Skin Cancer Unit of the Dermatology Department, Medical Faculty, University DuisburgEssen, West German Cancer Center, Hufelandstrasse 55, Essen 45147, Germany. E-mail: iris.helfrich@uk-essen.de

How to cite this article: Mörchen B, Shkura O, Stoll R, Helfrich I. Targeting the "undruggable" RAS - new strategies - new hope? Cancer Drug Resist 2019;2:813-26. http://dx.doi.org/10.20517/cdr.2019.21

Received: 1 Apr 2019 First Decision: 14 May 2019 Revised: 25 May 2019 Accepted: 4 Jun 2019 Published: 19 Sep 2019

Science Editor: Helen M. Coley Copy Editor: Cai-Hong Wang Production Editor: Jing Yu

\begin{abstract}
$K-R A S$ is the most frequently mutated oncogene in solid tumors, such as pancreatic, colon or lung cancer. The GTPase K-RAS can either be in an active (GTP-loaded) or inactive (GDP-loaded) form. In its active form K-RAS forwards signals from growth factors, cytokines or hormones to the nucleus, regulating essential pathways, such as cell proliferation and differentiation. In turn, activating somatic mutations of this proto-oncogene deregulate the complex interplay between GAP (GTPase-activating) - and GEF (Guanine nucleotide exchange factor) - proteins, driving neoplastic transformation. Due to a rather shallow surface, K-RAS lacks proper binding pockets for small molecules, hindering drug development over the past thirty years. This review summarizes recent progress in the development of low molecular antagonists and further shows insights of a newly described interaction between mutant K-RAS signaling and PD-L1 induced immunosuppression, giving new hope for future treatments of $K-R A S$ mutated cancer.
\end{abstract}

Keywords: K-RAS, small molecules, immune checkpoints, PD-1, PD-L1

\section{INTRODUCTION}

During evolution, cells have acquired the ability to respond to external stimuli through fine-tuned signaling cascades. To this extent, rat sarcoma (RAS)-proteins are small monomeric G-proteins that utilize various 


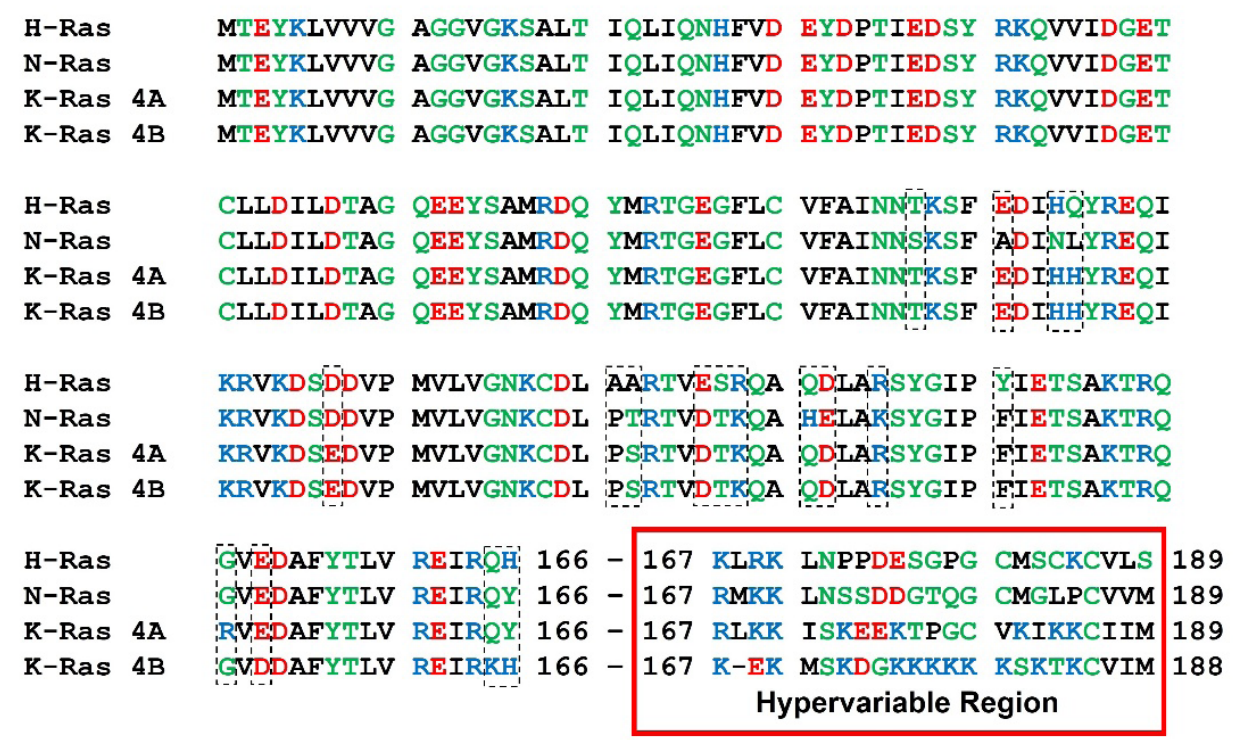

Figure 1. Amino acid sequences of RAS isoforms. The sequences of the RAS isoforms are compared and non-identical areas are marked by dashed rectangles. Amino acids 1-166 constitute the catalytic domain and amino acids 167-188/189 represent the hypervariable region. The amino acids are coloured based on their physico-chemical properties. Hydrophobic (black), polar/neutral (green), acidic (red) und basic (blue)

signal transduction pathways to regulate cell growth, differentiation, and apoptosis ${ }^{[1]}$. Due to their protooncogenic character, RAS proteins are a popular target in the field of cancer research ${ }^{[2]}$. The most prominent members of the RAS family are the isoforms N-RAS, H-RAS, K-RAS 4A and K-RAS 4B. These proteins emerge from proto-oncogenes, as their point-mutations are frequently present in different types of cancer ${ }^{[3]}$. The amino acid sequence of all four isoforms is shown in Figure 1. The first 166 amino acids constitute the catalytic G-domain and share a sequence identity of $89 \%$. The subsequent region spanning residues 167 to 188 and 189, respectively, differ among the isoforms. Hence, this C-terminal domain of RAS proteins is denoted as the hypervariable region.

K-RAS $4 \mathrm{~A}$ and $4 \mathrm{~B}$ represent two splicing variants of the exon 4 of the K-RAS pre-mRNA and all four isoforms possess the C-terminal CaaX-Box. The cysteine at position 185 or 186 respectively is followed by two aliphatic amino acids and it is this cysteine that plays a crucial role in the post-translational processing of RAS. All RAS isoforms are farnesylated to enable their anchoring to the membrane. Additionally, K-RAS 4A can be palmitoylated at cysteine 180, N-RAS at cysteine 181, and H-RAS at cysteine 181 and 184. This additional palmitoylation increases the hydrophobicity of the protein. In contrast to this, K-RAS $4 \mathrm{~B}$ is not palmitoylated but contains a poly lysine stretch (K175-K180) in the hypervariable region instead. This positively charged sequence facilitates a more transient anchorage to the negatively charged plasma membrane.

\section{RAS - function and structure}

RAS proteins function as binary molecular switches in signal transduction. They exist in an active GTPbound "ON"-state and an inactive GDP-bound "OFF"-state ${ }^{[4]}$. In the active state, the G-protein is able to bind to different effector proteins and transfer the signal. The intrinsic GTPase property enables the hydrolysis of GTP to GDP and hence the transition from the active to the inactive state. For the reactivation of RAS, the GDP nucleotide has to dissociate and the GTP nucleotide, which is present in a 10-fold excess, has to be incorporated. The intrinsic GDP-dissociation rate is $10^{-5} \mathrm{~s}^{-1[5]}$ and thus too low to be biologically significant. This rate is enhanced $10^{4}$-fold by guanine nucleotide exchange factors $(\mathrm{GEFs})^{[6]}$. These GEFs are positive regulators of the RAS activity. Hitherto, the protein encoded by the cell division cycle gene 25 (Cdc25) and the son of sevenless (SOS) are the best understood GEFs for RAS. GTPase activating proteins (GAPs) 


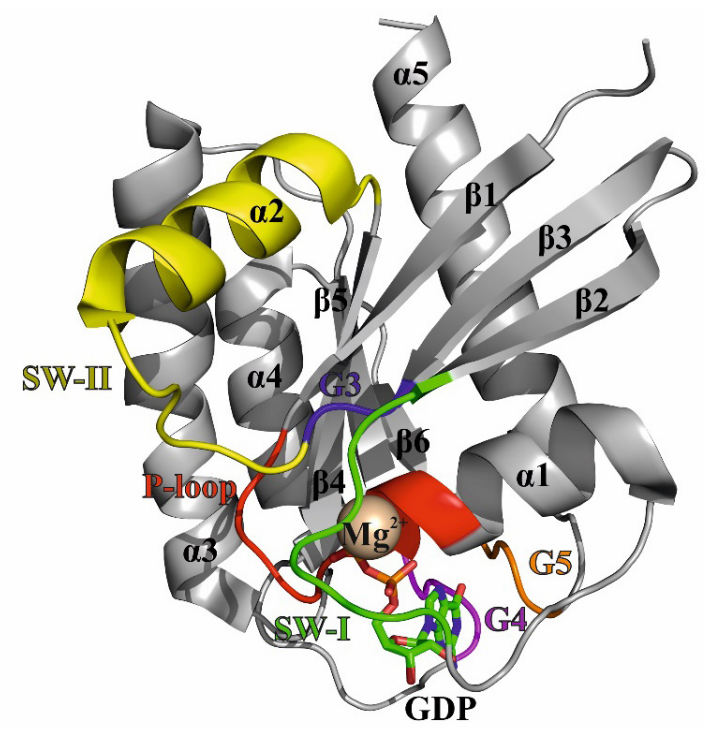

Figure 2. Crystal structure of K-RAS 4B GDP in ribbon representation (4EPY). The $\alpha$-helices ( $\alpha 1-\alpha 5)$, $\beta$-sheets ( $\beta 1-\beta 6$ ), the magnesiumion $\left(\mathrm{Mg}^{2+}\right)$, and the GDP nucleotide are labeled in black. The loops and switch regions are color-coded as follows: P-loop (red); switch I SW-I (green); G3-loop (blue); switch II - SW-II (yellow); G4-loop (magenta); G5-loop (orange)

function as counterparts to GEFs. They are essential for the hydrolysis of GTP as the inherent GTPase activity of RAS is only $10^{-4} \mathrm{~s}^{-1}$, which is amplified by GAPs by $10^{3}$-times.

The three-dimensional structure of RAS proteins explains the molecular mechanism of their biological function as cellular protein switches [Figure 2]. The guanosine nucleotide binds to the G-protein conserved G-domain. The G-domain constitutes of six $\beta$-sheets and five surrounding $\alpha$-helices which are connected with ten loops ${ }^{[6]}$.

Five of the ten loops that can be found between the secondary structural motifs are denoted as G1 to G5 motifs and participate in nucleotide binding. The G1-loop is glycine-rich region and utilizes the positive charge of lysine 16 to bind the $\beta$-phosphate of the nucleotide. The $\beta$-phosphate is additionally coordinated by serine 17 and a magnesium ion. The positive charge of magnesium is essential to stabilize the three negative phosphate groups of the nucleotide and, therefore, the G1-loop has also been described as the P-loop (GxxxGKS/T). The amino acids 32 to 38 form the G2-loop, also known as switch I. The threonine at position 35 is crucial in the binding of the $\gamma$-phosphate and the magnesium ion. In the presence of the $\gamma$-phosphate, the switch I region adopts the active conformation and participates in the effector binding ${ }^{[4]}$. The G3-loop includes the motif DxxG and shares glycine 60 with the neighboring switch II region, which comprises amino acids 60 to 76 . The glycine 60 is the second amino acid to bind the $\gamma$-phosphate through a hydrogen bond. Like switch I, switch II changes its conformation upon binding of GTP and does not only take part in effector binding but also acts as an interaction site for GEFs and GAPs ${ }^{[7]}$. The binding of the guanosine nucleotide is established by the G4-loop (N/TKxD aa 116 to 119) as well as the G5-loop (SAK aa 145 to 147$)^{[8]}$.

The conformational change of the protein from the inactive, GDP-bound state to the active, GTP-bound state has been coined as the loaded-spring mechanism ${ }^{[8]}$. In the active state, amino groups of threonine 35 (located in switch I) and glycine 60 (located in switch II) coordinate one oxygen atom of the $\gamma$-phosphate via hydrogen bonds. Upon hydrolysis of the $\gamma$-phosphate, the hydrogen bonds are broken and the flexible switch-loops spring back into their initial positions. Noteworthy, the loaded spring of the active state is not simply a rigid construct. The active state contains further two (sub-) states ${ }^{[\rho]}$. The difference between these two active states is the flexibility of the switches regions and the results in different affinities towards the 
effector proteins. In state 1 , the switch regions exhibit a higher conformational fluctuation compared to state 2 , which results in impaired binding to the effector $\mathrm{RAF}^{[10]}$. These conformational changes between the active and inactive conformation as well as the subdivision in active state 1 and 2 further emphasizes the dynamic nature of these switch regions of the RAS protein, an attribute referred to as polysterism ${ }^{[1]]}$.

\section{K-RAS IN CANCER}

\section{K-RAS signaling}

K-RAS acts as a cellular switch to regulate essential intrinsic pathways ${ }^{[12-14]}$. Extracellular stimuli, e.g., binding of growth factors, hormones or cytokines, are leading to a dimerization of receptor tyrosine kinases, which in turn activate GEFs, such as SOS, which leads to a nucleotide exchange and an activation of K-RAS. In its wild-type (wt) state K-RAS forwards signals from growth factors, cytokines or hormones to the nucleus, thereby regulating crucial pathways such as proliferation, differentiation and cell growth ${ }^{[15,16]}$. GAPs are responsible to enhance the catalytic rate of K-RAS GTPase function, leading to the hydrolyzation of GTP, the replacement with GDP, resulting in the inactivation of K-RAS ${ }^{[6,17]}$. Activating somatic mutations of this proto-oncogene disrupt this complex interplay between GEFs and GAPs ${ }^{[18,19]}$. GAPs can no longer increase the GTP hydrolyzation of oncogenic K-RAS, leading to a constitutive active mutant (mut) form driving neoplastic transformations in many cancer entities ${ }^{[20-22]}$. With a frequency of $21.6 \%$ somatic point mutations in $K-R A S$ are the most common mutations in the RAS gene of all human cancers, followed by an $8.0 \%$ incidence of N-RAS mutations and a $3.3 \%$ rate of $H-R A S$ mutations ${ }^{[23]}$.

The mitogen-activated protein kinase (MAPK) signaling cascade is crucial for the regulation of cellular functions like differentiation and cell growth of normal cells. Constitutive activation of this pathway leads to uncontrolled cell proliferation, transformation, dissemination of cancer cells and is one of the major effector pathways deregulated in K-RAS mutant cancer ${ }^{[24,25]}$. The pathway consists of the kinases RAF, mitogenactivated protein kinase/extracellular receptor-stimulated kinase 1/2 (MEK), and extracellular receptorstimulated kinase 1/2 (ERK), that are part of a phosphorylation cascade, downstream of activated GTP-bound RAS [Figure 3]. Once activated in the cytoplasm, ERK can activate proteins of the dynamic cytoskeletal complex that affect cell adhesion, trafficking, and movement ${ }^{[26,27]}$. In addition, it can also enter the nucleus and regulate various mitogenic transcription factors involved in stimulating cell proliferation ${ }^{[28,29]}$. The phosphoinositide 3-kinase (PI3K)/AKT pathway is the second major effector pathway downstream of mut $K-R A S$ signaling. GTP-bound K-RAS can recruit and phosphorylate PI3K. Activated PI3K can phosphorylate the downstream serine/threonine kinase AKT, which in turn translocate from the plasma membrane to other cellular compartments, phosphorylating target proteins responsible for regulating cell growth, cell survival and entry of the cell cycle ${ }^{[28,30]}$. An important downstream target of AKT is the mammalian target of rapamycin (mTOR) complex 1, which results in lipid or nucleotide synthesis, as well as in protein translation ${ }^{[31]}$.

Nowadays, cancer therapy is able to inhibit both effector pathways downstream of K-RAS via selective MEK(Binimetinib, Cobimetinib, Selumetinib, Trametinib) ${ }^{[32-36]}$, B-RAF- (Dabrafenib, Vemurafenib, Sorafenib, Encorafenib) ${ }^{[32,37-40]}$, or PI3K- (Idealisib, Buparlisib) ${ }^{[41-44]}$ inhibitors. However, continuous efforts over the past decades failed to develop novel therapies for $K-R A S$ mutant cancer.

\section{K-RAS mutations as a key molecule driving chemoresistance}

As there is no targeted therapy against $K-R A S$ mut cancers available today, chemotherapy is still standard care in the treatment of cancer patients harboring mutations in this very oncogene. Pancreatic cancer, associated with a $90 \%$ chance of mutations in the K-RAS gene, is very poorly responsive to standard care first and second line gemcitabine/fluoropyrimidine chemotherapy due to metabolism-dependent drug resistances ${ }^{[45,46]}$. Metabolic reprogramming of $K-R A S$ mut cancers is characterized by boosted glycolysis, glutaminolysis and 


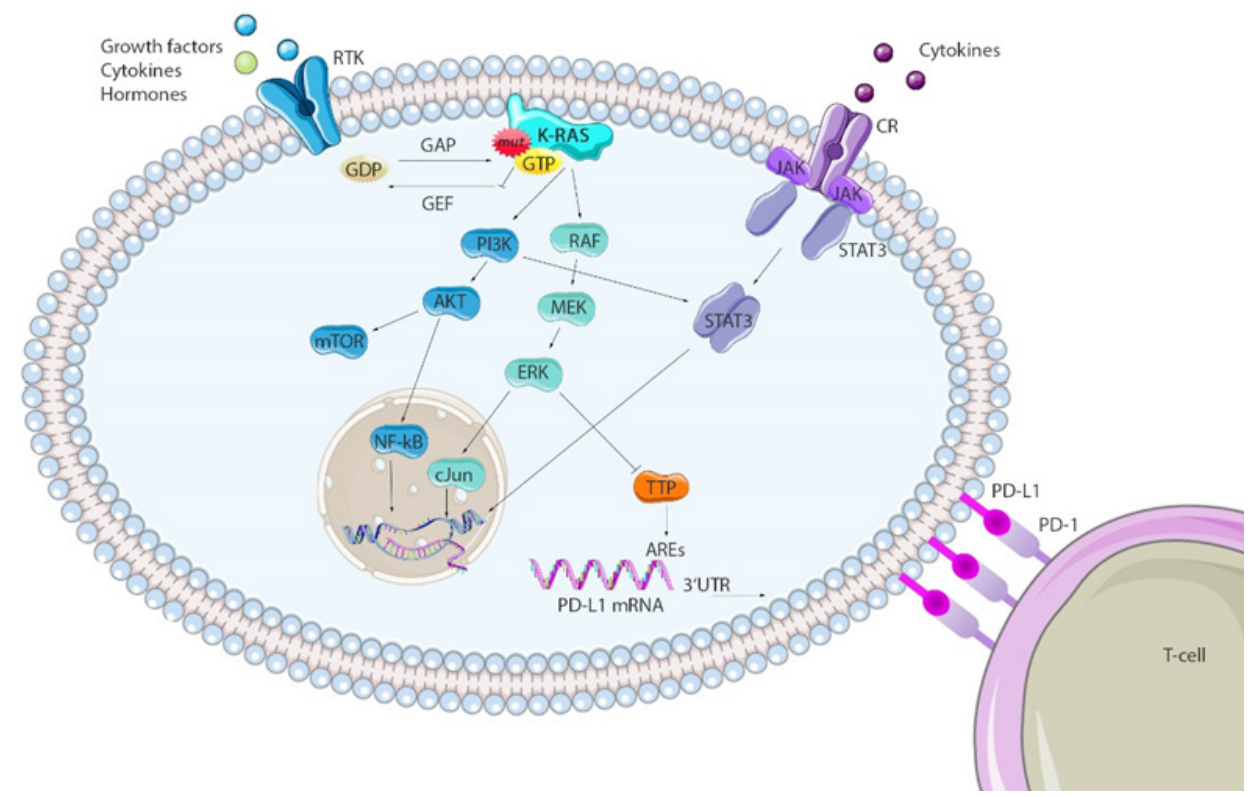

Figure 3. Major effector pathways of K-RAS and potential interactors. Mutations in K-RAS genes lead to constitutive GTP bound K-RAS proteins that activate downstream phosphorylation cascades. Major effector pathways dysregulated in $K-R A S$ mut cancers are the MAPK (RAF/MEK/ERK) and the PI3K/AKT pathway. Transcription factors regulated by these pathways, as well as STAT3, can bind to the $P D-\angle 1$ gene, enhancing the transcription rate. TPP destabilizes PD-L1 mRNA at AREs on the 3'UTR region and can be inhibited via MEK signaling in K-RAS mut cancers, prolonging the PD-L1 mRNA's half-life. This figure was partly created using SMART Servier Medical Art. ARE: AUrich elements; CR: cytokine receptor; RTK: receptor tyrosine kinase; TTP: tristetraprolin

pentose phosphate pathway, among others. Enhanced metabolism is not only providing more energy to the tumor, but has further impact on cell growth, rapid proliferation, invasion and drug resistance ${ }^{[47]}$. K-RAS and MYC oncogenes, as well as tumor suppressors like tumor protein p53, or phosphatase and tensin homolog (PTEN) are directly reprogramming a cancer cell's metabolism in order to sustain unrestricted tumor growth, thereby creating a metabolism addiction ${ }^{[47-49]}$. The correlation between the strong addiction of cells to this oncogene and its impact on drug resistance highlights the big need to find specific K-RAS antagonists for more efficient future therapies.

\section{VERY RECENT ADVANCES IN DEVELOPING LOW MOLECULAR WEIGHT ANTAGONISTS OF RAS}

RAS proteins already were in the focus of medicinal chemical endeavors in the last millennium but unfortunately proved to be difficult targets. This previously earned RAS the inglorious title of being "undruggable". During the past decade, tremendous progress has been made in modulating RAS by low molecular weight compounds, rendering RAS now indeed "druggable". In principle, there are three common approaches to address RAS directly. These include to target the CaaX-Box, the nucleotide binding domain (NBD) or a shallow hydrophobic pocket including the switches I and II.

\section{The CaaX-Box of RAS as a conceptionally new target site}

The first attempts to target the C-terminal CaaX-Box were based on the idea of interrupting the membrane binding and hence abolishing the signal transduction of RAS. The farnesyltransferase (FTase) is responsible for the farnesylation of all RAS isoforms at cysteine 185/186. The development of FTase inhibitors led to various products, of which some have entered clinical trials. Probably the most promising one is Tipifarnib that is currently in clinical trials ${ }^{[50]}$. However, studies have shown that Tipifarnib and other FTase inhibitors only affect the isoform H-RAS. The other isoforms, N-RAS as well as K-RAS $4 \mathrm{~A}$ and $4 \mathrm{~B}$, are still prenylated by geranylgeranyl transferase ${ }^{[51]}$. 


\section{The G12C RAS mutant as a special target}

Targeting the NBD is difficult due to the high picomolar affinity of RAS towards the nucleotide GDP and $\mathrm{GTP}^{[52]}$, as well as the high micromolar abundance of intracellular GDP and $\mathrm{GTP}^{[53]}$. One opportunity to compete with these conditions unveils itself in form of the oncogenic mutant $K-R A S$ G12C. The mutation of glycine 12 to cysteine establishes a nucleophilic target in proximity to the NBD for covalent binding of electrophilic inhibitors. SML-8-73-1 is a GDP-analogue which utilizes an electrophilic warhead to tether cysteine 12 and bind to the nucleotide binding pocket of $K-R A S^{[54]}$. As a GDP-analogue it is able to lock $K-R A S \mathrm{G} 12 \mathrm{C}$ in the inactive state. Another way to employ the solvent exposed cysteine anchor is based on the combination of an electrophile with a compound which is able to bind to the neighboring switch II region $^{[5,56]}$. The crucial aspect of the interaction with the switch II is that the binding pocket is only present in the inactive GDP-bound state. In case of the covalent inhibitor 12 developed by Ostrem et al. ${ }^{[56]}$ the binding to switch II results in disorder of both switch regions and enhances the affinity of K-RAS G12C towards GDP, hence rendering the protein inactive and decreasing the affinity towards the effector RAF. As the covalent tethering only works for the $\mathrm{G} 12 \mathrm{C}$ oncogene mutation but not for the other oncogene mutants such as $\mathrm{G} 12 \mathrm{~V}$, G12D and Q61H the demand for allosteric based inhibitors binding to the RAS-surface becomes evident.

\section{Targeting the hydrophobic ligand binding site located next to the switch regions of RAS}

Pharmaceuticals aiming at the surface of RAS have been extensively studied and different compounds have been synthesized that target the GDP or GTP form. Various peptidomimetics ${ }^{[57]}$ and antibody fragments ${ }^{[58]}$ have been characterized as possible antagonists for RAS based tumors. This review however, will focus on low-molecular weight compounds for RAS. The ligands discussed in the following chapter are presented in Figure 4 and are numbered according to the order in this figure.

In 2012, two groups, one from Genentech and the other from Vanderbilt University, independently published the discovery of ligands that bind reversibly to a shallow hydrophobic pocket located next to switch I and II in the GDP-bound state of $K-R A S 4 \mathrm{~B}^{[59,60]}$. For both compounds, (1) and (2) the interaction site overlaps with the GEF binding site and hence interferes with the RAS-GEF complex formation. In addition, Schöpel et al. ${ }^{[61,66]}$ reported bisphenol derivatives to bind to the same hydrophobic binding pocket in the sub-millimolar to micromolar range. Bisphenol A (BPA) is widely used as a plasticizer best known for its endocrine disrupting character ${ }^{[67]}$. Notably, while BPA antagonizes the interaction between RAS and SOS, the fluorinated bisphenols BPAF and $\mathrm{BPNH}_{2}$ (3) even induce an allosteric activation of the GDP-bound $K-R A S 4 \mathrm{~B}$. This induces a transition of RAS from the inactive, i.e., OFF-state, to a state in-between the OFFand $\mathrm{ON}$-state that is active ${ }^{[61]}$.

In 2017, Xie et al. ${ }^{[62]}$ reported on another ligand (4), that interacts with the hydrophobic binding pocket with the potential to hamper the binding of GTP. Further studies with non-small cell lung cancer (NSCLC) cell lines have shown that these compounds induce apoptosis in cell lines with oncogenic mutants of $K-R A S 4 \mathrm{~B}$ but not in wt $K$-RAS $4 \mathrm{~B}$ cell lines.

Modulating the transition from inactive to active RAS is a reasonable strategy in disrupting the signal transition, but so is the interruption of the RAS-Effector complex of the active GTP-bound form. Molecular Dynamic simulations of the switch regions have identified a cluster of small binding domains which are present in all RAS isoforms ${ }^{[63]}$. Welsch et al. ${ }^{[63]}$ developed a so-called Pan-RAS inhibitor (5), which shows micromolar affinity in vitro for the RAS isoforms K-RAS, H-RAS, and N-RAS in the active state. This compound was tested in in vivo experiments using xenograft mouse tumor models and was shown to inhibit tumor growth over a period of 15 days of treatment. Notably, this study also reports certain levels of toxicity as well as off-target activity, which could hamper the indicated tumor growth inhibition.

As mentioned above, antibody fragment-based therapeutics have already been tested (Antibody 1998), but prove to be challenging regarding their permeability into the cell. Based on the structural information of the 


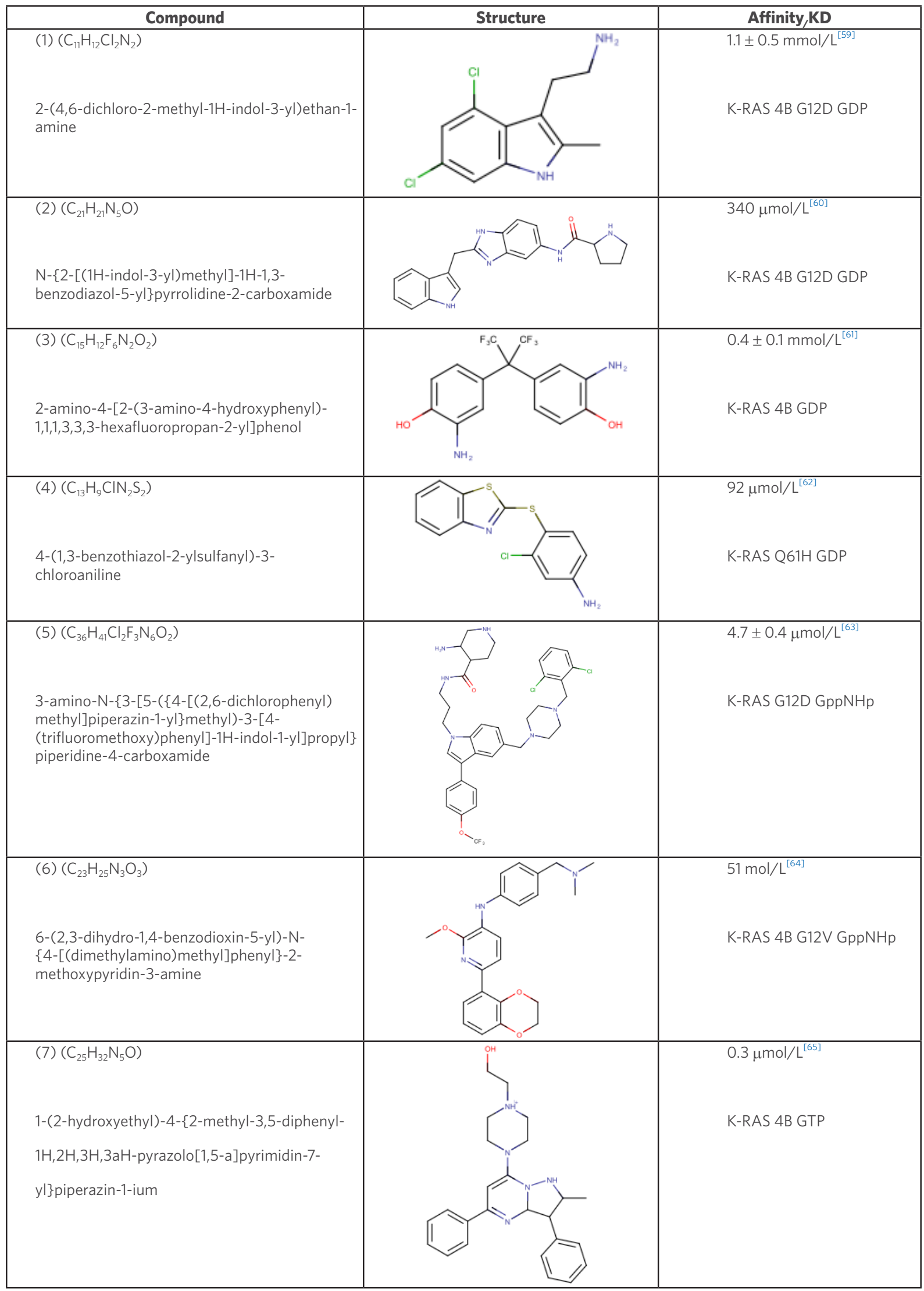

Figure 4. Low-molecular weight compounds targeting the RAS hydrophobic binding pocket 


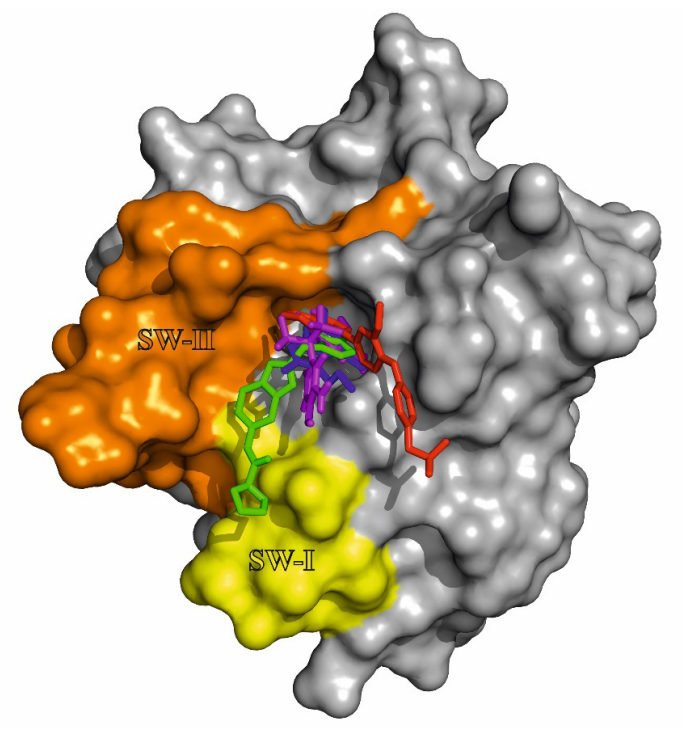

Figure 5. Ligand orientation in the hydrophobic binding pocket of K-RAS 4B. Co-crystal structure of (2) with K-RAS G12V (aa 1-169) [4EPY] in surface projection. The co-crystal structure of (1) [4DST] and (6) [6FA4] as well as (3) docked to [4DSO] have been aligned to 4EPY. The compounds are color-coded as follows: (1) blue; (2) green; (3) magenta; (6) red. The switch regions adjacent to the hydrophobic binding pocket are labeled switch I SW-I (yellow) and switch II SW-II (orange)

interaction between the antibody and its target site, Quevedo et al. ${ }^{[64]}$ have generated a low-molecular weight compound, Abd-7 (6), which targets the same region with nanomolar affinity. Abd-7 binds to the switch regions and antagonizes the protein-protein interaction between RAS and its effectors. Abd-7 interacts with $K-R A S \mathrm{G} 12 \mathrm{~V}$, which was bound to a non-hydrolysable GTP analogue, with an affinity of $51 \mathrm{nmol} / \mathrm{L}$. However, its IC $\mathrm{I}_{50}$ in cells is only $8 \mu \mathrm{mol} / \mathrm{L}$ that could suggest off-target effects and, once again, highlights the difference between molecular affinity in vitro and biological potency in vivo.

Finally, a novel inhibitor (7), by McCarthy et al. ${ }^{[65]}$ selectively interacts with both the GTP-bound wt and oncogenic mutants of K-RAS with sub-micromolar affinity but neither binds to the isoform H-RAS nor $\mathrm{N}-\mathrm{RAS}^{[65]}$. The binding region of this pyrazolopyrimidine-inhibitor overlaps with the switch regions and thereby enables the inhibition of the RAS-RAF complex. The differentiation between the active and inactive state of RAS targets presents a promising opportunity for the future development of much needed selective inhibitors of activating oncogenic RAS-mutations, such as G12V, G12D, and Q61H.

As described in the previous chapter, the presented ligands all bind to a hydrophobic binding pocket adjacent to the switch I and switch II region. To illustrate the binding orientation of the ligands the cocrystal structure of (2), [4EPY] was used in a surface projection. The co-crystal structures of (1) [4DST] and (6) [6FA4] as well as (3) NMR data-based docked onto $4 \mathrm{DSO}^{[61]}$ were then aligned to $4 \mathrm{EPY}$ [Figure 5].

\section{INFLUENCE OF ONCOGENIC K-RAS ON CANCER IMMUNOTHERPY}

\section{The programmed cell death protein 1 and its ligands}

Evidence that inflammatory triggers play a critical role in the development and progression of cancer was already emerging at the beginning of the 20th century. Cancer patients were infected with bacteria to provoke inflammation which also affected the tumor cells ${ }^{[68]}$. For example, until today non-muscle invasive bladder cancer patients are receiving a therapy based on the infection of the bladder with bacillus-calmette-guérin ${ }^{[69]}$.

In 2018, the Nobel Prize for medicine and physiology was dedicated to the US American immunologist James Allison and the Japanese immunologist Tasku Honjo for their discovery of so called "immune checkpoints" 
and their therapeutic "mode of action" as negative immune regulators. Programmed cell death protein 1 (PD-1), also known as CD279, discovered in 1992 by Honjo et al. ${ }^{[70]}$ is a transmembrane immunoinhibitory receptor of the CD28 family, mostly expressed on active T-cells. Binding of PD-1 with its ligands (PD-L1/ L2; known as CD274 and CD273) transmits a co-inhibitory signal in activated T-cells that promotes T-cell exhaustion, leading to tumor immune evasion ${ }^{[71]}$. However, contradictory results have suggested that PDL2 can serve as a negative and a positive regulator of $\mathrm{T}$ cell function. In 2004, Honjo et al. ${ }^{[72]}$ also discovered expression of PD-L1 on tumor cells, a potential strategy to suppress the host immune response and escape immune destruction. By using different preclinical models for melanoma and colon cancer he could show that a genetically or antibody-based PD-1 depletion leads to diminished hematogenous dissemination ${ }^{[72]}$. These experiments were the basis of the second immunotherapy approach of blocking PD-1/PD-L1 interactions. The anti-PD-1 antibody Nivolumab, in 2014 FDA approved for the treatment of advanced melanoma patients, is by the end of 2017and after more than 500 conducted clinical trials now approved for the treatment of nine different tumor entities including e.g., NSCLC, hodgkin lymphoma, squamous head and neck cancer or urothelial cancer ${ }^{[73]}$. Further FDA approved agents targeting the PD-1/PD-L1 axis e.g., Pembrolizumab (PD-1 inhibitor), Atezolizumab and Avelumab (both targeting PD-L1) are additionally as mono- or combination therapy in clinical use.

\section{Regulation of PD-L1 expression via oncogenic RAS signaling}

Hanahan and Weinberg ${ }^{[7]}$ defined the capability of cancer cells to suppress the immune system, and to evade immune responses/destruction as a hallmark of cancer $^{[74]}$. Moreover, the gain of mutations in cancer cells leads to changes in expression of surface molecules on the plasma membrane, so called neoantigens. The immune system is capable to recognize these neoantigens driving cytotoxic T-cells to destroy cancer cells. The immune system has certain breaks to dim or block this immune reaction, a mechanism preventing severe autoimmune responses, known as immune exhaustion ${ }^{[75]}$. As $K-R A S$ mutant cancer is characterized by a high presentation of neoantigens that should activate T-cell response, these tumors have the potential to escape from immune destruction. Since therapeutic monoclonal antibodies targeting the immune checkpoints was a breakthrough by demonstrating long-term survival benefit for some patients with solid cancer, recent evidence suggest that there might be a connection between the regulation of the PD1/PD-L1 axis and $K-R A S$ signaling.

Recent progress in developing small molecules against the formerly deemed "undruggable" $K-R A S$ gives hope to patients suffering from K-RAS mutant cancer. However, there are still no clinically active drugs against mutant $K-R A S$ variants available. Thus, understanding the complex interplay of oncogenic signaling and the immune system may help to circumvent mechanisms of resistance to immune checkpoint inhibitors. Preclinical and clinical data assumed that upregulation of PD-L1 on the cell surface of tumor cells seems to be a major driver of immune evasion. It was reported, that K-RAS mut NSCLC patients showed a prolonged overall survival after a Nivolumab treatment compared to $K-R A S$ wt patients, who did not benefit from a PD-1 blockade ${ }^{[7]}$. Moreover, Garon et al. ${ }^{[76]}$ recently reported increased PD-L1 expression in K-RAS mut vs. K-RAS wt NSCLC tumors ${ }^{[7]}$. Therefore, it is of major importance to unravel the molecular mechanisms involved in the reciprocal interaction of activated $K-R A S$ and presentation of PD-L1.

The best characterized stimulus from the tumor microenvironment that leads to up-regulation of PD-L1 expression is the inflammatory cytokine interferon (IFN) $\gamma$, acting via activation of the Janus kinase (JAK)/STAT pathway ${ }^{[78]}$. It has been shown that STAT3 binds to the PD-L1 promotor region, enhancing its transcription in vitro and in vivo ${ }^{[79]}$. This mechanism was proven in anaplastic large-cell lymphoma, T-cell lymphoma and in K-RAS mut NSCLC ${ }^{[79-81]}$. Recent evidence suggests that oncogenic RAS signaling can directly increase PD-L1 mRNA stability, which leads to enhanced PD-L1 surface expression and impaired tumor immune surveillance $^{[82]}$. Whereas PD-L1 protein level were significantly increased in BRAF V600E mut melanoma cell lines resistant to BRAF inhibition, this effect was decreased both after using small interfering RNA (siRNA) 
mediated knockdown of ERK1/2, and after pharmacologic inhibition of MEK ${ }^{[83,84]}$. Jiang et al. ${ }^{[83]}$ further showed that depletion of c-Jun (transcription factor downstream of ERK) and STAT3 resulted in a synergistic decrease of PD-L1 surface expression, indicating a cooperation of RAS and STAT3 signaling in controlling PD-L1 expression in melanoma. MEK and ERK abrogation was further effective to minimize ectopic PD-L1 expression in K-RAS mut NSCLC cell lines ${ }^{[81]}$.

Moreover, the second major effector pathway of RAS, the PI3K/AKT pathway, also seems to play a role in regulating PD-L1 expression, as inhibition of either AKT or mTOR resulted in decreased PD-L1 levels in triple negative breast cancer (TNBC) ${ }^{[85]}$. This is in line with the observation that a knockdown of the PTEN (negative regulator of $\mathrm{P} 13 \mathrm{~K}$ ) leads to elevated PD-L1 expression level in the same TNBC cell model, as well as in a murine lung squamous cell carcinoma model ${ }^{[86]}$. Inhibition of PI3K resulted in PD-L1 down-regulation in many tumor entities including K-RAS or EGFR mut NSCLC, renal cell carcinoma and melanoma ${ }^{[83,87,88]}$.

Coelho et al. ${ }^{[82]}$ were the first describing a novel translational control mechanism of PD-L1 expression via direct mut K-RAS signaling. By using a tamoxifen-inducible ER-KRAS ${ }^{\mathrm{G} 12 \mathrm{~V}}$ fusion protein they could show a profound up-regulation of PD-L1 mRNA and surface protein level upon activation of oncogenic RAS. In contrast to IFN- $\gamma$ dependent PD-L1 regulation, induction of PD-L1 via activated RAS is mediated by an increased half-life of the PD-L1 mRNA ${ }^{[82]}$. AU-rich elements (AREs) of $3^{\prime}$ untranslated regions (UTR) of the PD-L1 mRNA are targets of the ARE-binding protein tristetraprolin (TTP). Once TTP is physically bound to AREs, it destabilizes the PD-L1 mRNA. It was proven in lung cancer cell lines, that mut K-RAS driven MEK signaling reduces intracellular TTP levels, thereby prolonging PD-L1mRNA's half-life and stability, which in turn increased the cells PD-L1 surface expression. Additionally, TTP restoration in K-RAS mut tumors resulted in enhanced anti-tumor immunity in murine colon cancer models ${ }^{[82]}$. Curiously, a TTP deficiency in macrophages and cytotoxic T-cells increases T-cell induced anti-tumor activities via modified cytokine expression ${ }^{[89]}$.

Interestingly, genomic alterations in the 3'UTR region of the PD-L1 gene itself have an impact on the PD-L1 expression in some cancers ${ }^{[00]}$, which may indicate a shared TTP regulation mechanism of the PD-L1 mRNA, as it is the case in K-RAS mut cancers.

\section{Clinical implication of $K-R A S$ status and PD-L1 expression in cancer}

Continuous efforts in the past three decades failed to develop approved therapies targeting activated $K-R A S$. Although it is too early to claim clinical benefits, new therapeutic strategies can evolve from the knowledge about the interaction of these key molecules in tumor biology. So, the strategy to combine immunotherapies has brought new hope for $K-R A S$-dependent tumors. A more recent clinical study analyzed the impact of the individual $K-R A S$ mutations on PD-L1 expression and clinical outcome in lung adenocarcinoma patients. They showed that exclusively $\mathrm{G} 12 \mathrm{~V}$, and none of the other mutations in $K-R A S$, leads to a significant increase in PD-L1 expression ${ }^{[91]}$. This study further states a correlation between high PD-L1 expression and improved OS only in K-RAS mut cancer. Additionally, Falk et al. ${ }^{[1]]}$ reported that high PD-L1 expressing immune cells in these patients correlated with poor OS, independent on the tumor's $K-R A S$ mutation. These findings show how heterogeneous $K-R A S$ mutational status affects the immune system in lung adenocarcinomas, indicating a $K-R A S$ dependent patient stratification for immunotherapy in future.

\section{Combination of small molecules and PD-1 inhibition in patients with RAS mutant tumors}

Currently there are many preclinical analyses and clinical trials ongoing to target $K-R A S$-driven cancer. A broad range of novel therapeutic strategies, e.g., the usage of siRNA and exosome siRNA, monoclonal antibodies targeting the GTP-bound $K-R A S$, use of oligonucleotides to prevent membrane embedding and farnesyltransferase inhibitors implicate new hope. However, it will take some time before we can draw conclusions about the effectiveness of these new therapeutic approaches. Nevertheless, a currently recruiting 
phase $1 \mathrm{~b}$ clinical trial, initiated by Targovax, is evaluating the effect of the multi-kinase inhibitor TG02 in combination with the anti-PD-1 antibody Pembrolizumab in patients with locally advanced primary tumors in RAS mutated colorectal cancers (ID: CT TG02-01). TG02, a second-generation RAS neo-antigen vaccine, selectively targets tyrosine kinase receptors (e.g., fms-like tyrosine kinase receptor-3), mitotic and transcriptional cyclin dependent kinases 1, 2, 3, 5, as well as lymphocyte-specific protein tyrosine kinase, proto-oncogene tyrosine-protein kinase Fyn, non-receptor tyrosine-protein kinase 2 and JAK $2^{[22,93]}$. TG02 is reported to induce apoptosis in cell lines and primary cells of acute myeloid leukemia, and lead to tumor regression in xenograft models ${ }^{[93]}$. In 2018, Su et al ${ }^{[94]}$ reported induction of apoptosis, reduced tumor cell proliferation and a prolonged OS of mice harboring glioblastoma after TG02 treatment. Early exploratory clinical data indicate that TG02 induces immune response in patients with so called "hot tumors" which are rich in numbers of activated tumor-infiltrating T-cells compared to controls. Moreover, PD-1 expression was observed in both circulating and tumor-infiltrating T-cells. This further strengthens the rationale for combining TG02 with PD-1 checkpoint blockade. Whether this dual targeting would be advantageous compared with the previous therapeutic strategies still await clinical validation.

\section{CONCLUSION}

$R A S$ oncogenes are often activated by point mutations in a wide range of different cancer entities, thereby identifying RAS as an important clinical target. There are three RAS genes (H-RAS, K-RAS and N-RAS) whereas $K-R A S$ is the most frequently mutated oncogene in cancer. Activating somatic mutations of this proto-oncogene occur in approximately $30 \%$ of human cancers driving neoplastic transformation. The $K-R A S$ protein is lacking proper binding pockets for small molecules, resulting in no progress in drug development over the past thirty years. Recently new findings e.g., the identification of a new binding pocket interfering with the SOS-K-RAS interaction has aroused the interest to re-visit the "formerly undruggable" target. In addition, more recent insights into the interplay between mutant RAS and the PD-1/PD-L1 signaling provide a portfolio of synergistic treatment options through the additional use of inhibitors targeting the immune checkpoints. Nevertheless, although novel therapeutic directions are currently under investigation, the risk for induction of acquired resistance is also in clinically effective drugs expected given by the strong selective pressure applied to the genetically unstable cancer cells.

\section{DECLARATIONS}

\section{Authors' contributions}

Writing and original draft preparation: Mörchen B, Shkura O, Stoll R, Helfrich I

Review and editing: Stoll R, Helfrich I

\section{Availability of data and materials}

Not applicable.

\section{Financial support and sponsorship}

This work was partially supported by the European Fond for Regional Development (EFRD) (EFRE-0800951) to Mörchen B and Helfrich I; and (EFRE-0800947) to Shkura O and Stoll R.

\section{Conflicts of interest}

All authors declared that there are no conflicts of interest.

\section{Ethical approval and consent to participate}

Not applicable. 


\section{Consent for publication}

Not applicable.

\section{Copyright}

(c) The Author(s) 2019.

\section{REFERENCES}

1. Feig LA, Buchsbaum RJ. Cell signaling: life or death decisions of Ras proteins. Curr Biol 2002;12:259-61.

2. Shields JM, Pruitt K, Shaub A, Der CJ. Understanding Ras : 'it ain’t over 'til it's over'. Trends Cell Biol 2000;10:147-54.

3. Bos JL. ras oncogenes in human cancer : a review. Cancer Res 1989;49:4682-9.

4. Vetter IR, Wittinghofer A. The guanine nucleotide - binding switch in three dimensions. Science 2001;294:1299-304.

5. Lenzen C, Cool RH, Prinz H, Wittinghofer A. Kinetic analysis by fluorescence of the interaction between Ras and the catalytic domain of the guanine nucleotide exchange factor Cdc25Mm. Biochemistry 1998;37:7420-30.

6. Karnoub AE, Weinberg RA. Ras oncogenes: split personalities. Nat Rev Mol Cell Biol 2008;9:517-31.

7. Vetter IR. The structure of the G domain of the Ras superfamily. Ras Superfamily Small G Proteins: Biology and Mechanisms 1. Wien: Springer Verlag; 2014. pp. 25-51.

8. Wittinghofer A, Vetter IR. Structure-function relationships of the G domain, a canonical switch motif. Ann Rev Biochem 2011;80:94371.

9. Spoerner M, Nuehs A, Herrmann C, Steiner G, Kalbitzer HR. Slow conformational dynamics of the guanine nucleotide-binding protein Ras complexed with the GTP analogue GTP $\gamma$ S. FEBS J 2007;274:1419-33.

10. Spoerner M, Hozsa C, Poetzl JA, Reiss K, Ganser P, et al. Conformational states of human rat sarcoma (Ras) protein complexed with its natural ligand GTP and their role for effector interaction and GTP hydrolysis. J Biol Chem 2010;285:39768-78.

11. Ito Y, Yamasaki K, Iwahara J, Terada T, Kamiya A, et al. Regional polysterism in the GTP-bound form of the human c-Ha-Ras protein. Biochemistry 1997;36:9109-19.

12. Barbacid M. ras genes. Annu Rev Biochem 1987;56:779-827.

13. Malumbres M, Barbacid M. RAS oncogenes: the first 30 years. Nat Rev Cancer 2003;3:459-65.

14. Vetter IR, Wittinghofer A. The guanine nucleotide-binding switch in three dimensions. Science 2001;294:1299-304.

15. Hancock JF. Ras proteins: different signals from different locations. Nat Rev Mol Cell Biol 2003;4:373-84.

16. Lemmon MA, Schlessinger J. Cell signaling by receptor tyrosine kinases. Cell 2010;141:1117-34.

17. Cox AD, Der CJ. Ras history: the saga continues. Small GTPases 2010;1:2-27.

18. Gibbs JB, Sigal IS, Poe M, Scolnick EM. Intrinsic GTPase activity distinguishes normal and oncogenic ras p21 molecules. Proc Natl Acad Sci U S A 1984;81:5704-8.

19. McGrath JP, Capon DJ, Goeddel DV, Levinson AD. Comparative biochemical properties of normal and activated human ras p21 protein. Nature 1984;310:644-9.

20. Sweet RW, Yokoyama S, Kamata T, Feramisco JR, Rosenberg M, et al. The product of ras is a GTPase and the T24 oncogenic mutant is deficient in this activity. Nature 1984;311:273-5.

21. Scheffzek K, Lautwein A, Kabsch W, Ahmadian MR, Wittinghofer A. Crystal structure of the GTPase-activating domain of human p120GAP and implications for the interaction with Ras. Nature 1996;384:591-6.

22. Schubbert S, Shannon K, Bollag G. Hyperactive Ras in developmental disorders and cancer. Nat Rev Cancer 2007;7:295-308.

23. Jemal A, Siegel R, Xu J, Ward E. Cancer statistics, 2010. CA Cancer J Clin 2010;60:277-300.

24. Dhillon AS, Hagan S, Rath O, Kolch W. MAP kinase signalling pathways in cancer. Oncogene 2007;26:3279-90.

25. De Luca A, Maiello MR, D'Alessio A, Pergameno M, Normanno N. The RAS/RAF/MEK/ERK and the PI3K/AKT signalling pathways: role in cancer pathogenesis and implications for therapeutic approaches. Expert Opin Ther Targets 2012;16 Suppl 2:S17-27.

26. Pullikuth AK, Catling AD. Scaffold mediated regulation of MAPK signaling and cytoskeletal dynamics: a perspective. Cell Signal 2007:19:1621-32.

27. Ma L, Chen Z, Erdjument-Bromage H, Tempst P, Pandolfi PP. Phosphorylation and functional inactivation of TSC2 by Erk implications for tuberous sclerosis and cancer pathogenesis. Cell 2005;121:179-93.

28. Choi M, Bien H, Mofunanya A, Powers S. Challenges in Ras therapeutics in pancreatic cancer. Semin Cancer Biol 2019;54:101-8

29. Yang SH, Sharrocks AD, Whitmarsh AJ. MAP kinase signalling cascades and transcriptional regulation. Gene 2013;513:1-13.

30. Andjelkovic M, Alessi DR, Meier R, Fernandez A, Lamb NJ, et al. Role of translocation in the activation and function of protein kinase B. J Biol Chem 1997;272:31515-24.

31. Cai SL, Tee AR, Short JD, Bergeron JM, Kim J, et al. Activity of TSC2 is inhibited by AKT-mediated phosphorylation and membrane partitioning. J Cell Biol 2006;173:279-89.

32. Long GV, Hauschild A, Santinami M, Atkinson V, Mandala M, et al. Adjuvant Dabrafenib plus Trametinib in stage III BRAF-mutated melanoma. N Engl J Med 2017;377:1813-23.

33. Janne PA, van den Heuvel MM, Barlesi F, Cobo M, Mazieres J, et al. Selumetinib plus docetaxel compared with docetaxel alone and progression-free survival in patients with KRAS-mutant advanced non-small cell lung cancer: the SELECT-1 randomized clinical trial. JAMA 2017;317:1844-53. 
34. Pulido JS, Heier JS, Marmorstein AD. Selumetinib in plexiform neurofibromas. N Engl J Med 2017;376:1195.

35. Signorelli J, Shah Gandhi A. Cobimetinib. Ann Pharmacother 2017;51:146-53.

36. Dummer R, Schadendorf D, Ascierto PA, Arance A, Dutriaux C, et al. Binimetinib versus dacarbazine in patients with advanced NRAS-mutant melanoma (NEMO): a multicentre, open-label, randomised, phase 3 trial. Lancet Oncol 2017;18:435-45.

37. Nijenhuis CM, Huitema AD, Blank C, Haanen JB, van Thienen JV, et al. Clinical Pharmacokinetics of Vemurafenib in BRAF-mutated melanoma patients. J Clin Pharmacol 2017;57:125-8.

38. Zhang W, Heinzmann D, Grippo JF. Clinical pharmacokinetics of vemurafenib. Clin Pharmacokinet 2017;56:1033-43.

39. Delord JP, Robert C, Nyakas M, McArthur GA, Kudchakar R, et al. Phase I dose-escalation and -expansion study of the BRAF inhibitor encorafenib (LGX818) in metastatic BRAF-mutant melanoma. Clin Cancer Res 2017;23:5339-48.

40. Gounder MM, Mahoney MR, Van Tine BA, Ravi V, Attia S, et al. Sorafenib for advanced and refractory desmoid tumors. N Engl J Med 2018;379:2417-28.

41. Di Leo A, Johnston S, Lee KS, Ciruelos E, Lonning PE, et al. Buparlisib plus fulvestrant in postmenopausal women with hormonereceptor-positive, HER2-negative, advanced breast cancer progressing on or after mTOR inhibition (BELLE-3): a randomised, doubleblind, placebo-controlled, phase 3 trial. Lancet Oncol 2018;19:87-100.

42. Furman RR, Sharman JP, Coutre SE, Cheson BD, Pagel JM, et al. Idelalisib and rituximab in relapsed chronic lymphocytic leukemia. N Engl J Med 2014;370:997-1007.

43. Gopal AK, Kahl BS, de Vos S, Wagner-Johnston ND, Schuster SJ, et al. PI3Kdelta inhibition by idelalisib in patients with relapsed indolent lymphoma. N Engl J Med 2014;370:1008-18.

44. Younes A, Salles G, Martinelli G, Bociek RG, Barrigon DC, et al. Pan-phosphatidylinositol 3-kinase inhibition with buparlisib in patients with relapsed or refractory non-Hodgkin lymphoma. Haematologica 2017;102:2104-12.

45. Wang C, Liu B, Xu X, Zhuang B, Li H, et al. Toward targeted therapy in chemotherapy-resistant pancreatic cancer with a smart triptolide nanomedicine. Oncotarget 2016;7:8360-72.

46. Takai E, Yachida S. Genomic alterations in pancreatic cancer and their relevance to therapy. World J Gastrointest Oncol 2015;7:250-8.

47. Grasso C, Jansen G, Giovannetti E. Drug resistance in pancreatic cancer: Impact of altered energy metabolism. Crit Rev Oncol Hematol 2017;114:139-52.

48. Hezel AF, Kimmelman AC, Stanger BZ, Bardeesy N, Depinho RA. Genetics and biology of pancreatic ductal adenocarcinoma. Genes Dev 2006;20:1218-49.

49. Cetinbas NM, Sudderth J, Harris RC, Cebeci A, Negri GL, et al. Glucose-dependent anaplerosis in cancer cells is required for cellular redox balance in the absence of glutamine. Sci Rep 2016;6:32606.

50. Soleimani A, Amirinejad M, Rahsepar S, Vazirian F, Bahrami A, et al. Therapeutic potential of RAS prenylation pharmacological inhibitors in the treatment of breast cancer, recent progress, and prospective. J Cell Biochem 2019;120:6860-7.

51. Whyte DB, Kirschmeier P, Hockenberry TN, Nunez-Oliva I, James L, et al. K- and N-Ras are geranylgeranylated in cells treated with farnesyl protein transferase inhibitors. J Biol Chem 1997;272:14459-64.

52. Feuerstein J, Kalbitzer HR, John J, Goody RS, Wittinghofer A. Characterisation of the metal-ion-GDP complex at the active sites of transforming and nontransforming p21 proteins by observation of the 17O-Mn superhyperfine coupling and by kinetic methods. Eur J Biochem 1987;162:49-55.

53. Traut TW. Physiological concentrations of purines and pyrimidines. Mol Cell Biochem 1994;140:1-22.

54. Lim SM, Westover KD, Ficarro SB, Harrison RA, Choi HG, et al. Therapeutic targeting of oncogenic K-Ras by a covalent catalytic site inhibitor. Angew Chem Int Ed Engl 2014;53:199-204.

55. Patricelli MP, Janes MR, Li LS, Hansen R, Peters U, et al. Selective inhibition of oncogenic KRAS output with small molecules targeting the inactive state. Cancer Discov 2016;6:316.

56. Ostrem JM, Peters U, Sos ML, Wells JA, Shokat KM. K-Ras(G12C) inhibitors allosterically control GTP affinity and effector interactions. Nature 2013;503:548-51.

57. Patgiri A, Yadav KK, Arora PS, Bar-sagi D. An orthosteric inhibitor of Ras-Sos interaction. Nat Chem Biol 2011;7:585-7.

58. Cochet O, Kenigsberg M, Delumeau I, Virone-Oddos A, Multon MC, et al. Intracellular Expression of an Antibody Fragmentneutralizing p21 Ras Promotes Tumor Regression. Cancer Research 1998;58:1170.

59. Maurer T, Garrenton LS, Oh A, Pitts K, Anderson DJ, et al. Small-molecule ligands bind to a distinct pocket in Ras and inhibit SOSmediated nucleotide exchange activity. Proc Natl Acad Sci U S A 2012;109:5299-304.

60. Sun Q, Burke JP, Phan J, Burns MC, Olejniczak ET, et al. Discovery of small molecules that bind to K-Ras and inhibit Sos-mediated activation. Angew Chem Int Ed Engl 2012;51:6140-3.

61. Schöpel M, Shkura O, Seidel J, Kock K, Zhong X, et al. Allosteric activation of GDP-bound ras isoforms by bisphenol derivative plasticisers. Int J Mol Sci 2018;19:1133.

62. Xie C, Li Y, Li LL, Fan XX, Wang YW, et al. Identification of a new potent inhibitor targeting KRAS in non-small cell lung cancer cells. Front Pharmacol 2017;8:823.

63. Welsch ME, Kaplan A, Chambers JM, Stokes ME, Bos PH, et al. Multivalent small-molecule Pan-RAS inhibitors. Cell 2017;168:878-89. e29.

64. Quevedo CE, Cruz-Migoni A, Bery N, Miller A, Tanaka T, et al. Small molecule inhibitors of RAS-effector protein interactions derived using an intracellular antibody fragment. Nature communications 2018;9:3169.

65. McCarthy MJ, Pagba CV, Prakash P, Naji AK, van der Hoeven D, et al. Discovery of high-affinity noncovalent allosteric KRAS inhibitors that disrupt effector binding. ACS Omega 2019;4:2921-30. 
66. Schöpel M, Jockers KFG, Düppe PM, Autzen J, Potheraveedu VN, et al. Bisphenol a binds to Ras proteins and competes with guanine nucleotide exchange: implications for GTPase-selective antagonists. J Med Chem 2013;56:9664-72.

67. Rogers JA, Metz L, Yong VW. Review: endocrine disrupting chemicals and immune responses: a focus on bisphenol-A and its potential mechanisms. Mol Immunol 2013;53:421-30.

68. Herr HW, Morales A. History of bacillus calmette-guerin and bladder cancer: an immunotherapy success story. J Urol 2008;179:53-6.

69. Balakrishnan AS, Washington SL 3rd, Meng MV, Porten SP. Determinants of guideline-based treatment in patients With cT1 bladder cancer. Clin Genitourin Cancer 2019;17:e461-71.

70. Ishida Y, Agata Y, Shibahara K, Honjo T. Induced expression of PD-1, a novel member of the immunoglobulin gene superfamily, upon programmed cell death. EMBO J 1992;11:3887-95.

71. Xia Y, Jeffrey Medeiros L, Young KH. Signaling pathway and dysregulation of PD1 and its ligands in lymphoid malignancies. Biochim Biophys Acta 2016;1865:58-71.

72. Iwai Y, Terawaki S, Honjo T. PD-1 blockade inhibits hematogenous spread of poorly immunogenic tumor cells by enhanced recruitment of effector T cells. Int Immunol 2005;17:133-44.

73. Gong J, Chehrazi-Raffle A, Reddi S, Salgia R. Development of PD-1 and PD-L1 inhibitors as a form of cancer immunotherapy: a comprehensive review of registration trials and future considerations. J Immunother Cancer 2018;6:8.

74. Hanahan D, Weinberg RA. Hallmarks of cancer: the next generation. Cell 2011;144:646-74.

75. McKinney EF, Smith KG. T cell exhaustion and immune-mediated disease-the potential for therapeutic exhaustion. Curr Opin Immunol 2016;43:74-80.

76. Garon EB, Rizvi NA, Hui R, Leighl N, Balmanoukian AS, et al. Pembrolizumab for the treatment of non-small-cell lung cancer. N Engl J Med 2015;372:2018-28.

77. Borghaei H, Paz-Ares L, Horn L, Spigel DR, Steins M, et al. Nivolumab versus docetaxel in advanced nonsquamous non-small-cell lung cancer. N Engl J Med 2015;373:1627-39.

78. Song TL, Nairismagi ML, Laurensia Y, Lim JQ, Tan J, et al. Oncogenic activation of the STAT3 pathway drives PD-L1 expression in natural killer/T-cell lymphoma. Blood 2018;132:1146-58.

79. Marzec M, Zhang Q, Goradia A, Raghunath PN, Liu X, et al. Oncogenic kinase NPM/ALK induces through STAT3 expression of immunosuppressive protein CD274 (PD-L1, B7-H1). Proc Natl Acad Sci U S A 2008;105:20852-7.

80. Atsaves V, Tsesmetzis N, Chioureas D, Kis L, Leventaki V, et al. PD-L1 is commonly expressed and transcriptionally regulated by STAT3 and MYC in ALK-negative anaplastic large-cell lymphoma. Leukemia 2017;31:1633-7.

81. Sumimoto H, Takano A, Teramoto K, Daigo Y. RAS-mitogen-activated protein kinase signal is required for enhanced PD-L1 expression in human lung cancers. PLoS One 2016;11:e0166626.

82. Coelho MA, de Carne Trecesson S, Rana S, Zecchin D, Moore C, et al. Oncogenic RAS signaling promotes tumor immunoresistance by stabilizing PD-L1 mRNA. Immunity 2017;47:1083-99.e6.

83. Jiang X, Zhou J, Giobbie-Hurder A, Wargo J, Hodi FS. The activation of MAPK in melanoma cells resistant to BRAF inhibition promotes PD-L1 expression that is reversible by MEK and PI3K inhibition. Clin Cancer Res 2013;19:598-609.

84. Sanlorenzo M, Vujic I, Floris A, Novelli M, Gammaitoni L, et al. BRAF and MEK inhibitors increase PD-1-positive melanoma cells leading to a potential lymphocyte-independent synergism with anti-PD-1 antibody. Clin Cancer Res 2018;24:3377-85.

85. Mittendorf EA, Philips AV, Meric-Bernstam F, Qiao N, Wu Y, et al. PD-L1 expression in triple-negative breast cancer. Cancer Immunol Res 2014;2:361-70.

86. Xu C, Fillmore CM, Koyama S, Wu H, Zhao Y, et al. Loss of Lkb1 and Pten leads to lung squamous cell carcinoma with elevated PD-L1 expression. Cancer Cell 2014;25:590-604.

87. Lastwika KJ, Wilson W 3rd, Li QK, Norris J, Xu H, et al. Control of PD-L1 Expression by oncogenic activation of the AKT-mTOR pathway in non-small cell lung cancer. Cancer Res 2016;76:227-38.

88. Balan M, Miery Teran E, Waaga-Gasser AM, Gasser M, Choueiri TK, et al. Novel roles of c-Met in the survival of renal cancer cells through the regulation of HO-1 and PD-L1 expression. J Biol Chem 2015;290:8110-20.

89. Wang Q, Ning H, Peng H, Wei L, Hou R, et al. Tristetraprolin inhibits macrophage IL-27-induced activation of antitumour cytotoxic T cell responses. Nat Commun 2017;8:867.

90. Kataoka K, Shiraishi Y, Takeda Y, Sakata S, Matsumoto M, et al. Aberrant PD-L1 expression through 3'-UTR disruption in multiple cancers. Nature 2016;534:402-6.

91. Falk AT, Yazbeck N, Guibert N, Chamorey E, Paquet A, et al. Effect of mutant variants of the KRAS gene on PD-L1 expression and on the immune microenvironment and association with clinical outcome in lung adenocarcinoma patients. Lung Cancer 2018;121:70-5.

92. Pallis M, Abdul-Aziz A, Burrows F, Seedhouse C, Grundy M, et al. The multi-kinase inhibitor TG02 overcomes signalling activation by survival factors to deplete MCL1 and XIAP and induce cell death in primary acute myeloid leukaemia cells. Br J Haematol 2012;159:191203.

93. Goh KC, Novotny-Diermayr V, Hart S, Ong LC, Loh YK, et al. TG02, a novel oral multi-kinase inhibitor of CDKs, JAK2 and FLT3 with potent anti-leukemic properties. Leukemia 2012;26:236-43.

94. Su YT, Chen R, Wang H, Song H, Zhang Q, et al. Novel targeting of transcription and metabolism in glioblastoma. Clin Cancer Res 2018;24:1124-37. 